\section{Designer meetings}

\author{
ROBERT I. LeCHLER', MegAN SYKeS ${ }^{2}$,ANgus W.ThOMSON ${ }^{3}$ \\ AND LAURENCE A.TURKA ${ }^{4}$
}

'Imperial College of Medicine, London, UK. ${ }^{2}$ Massachusetts General Hospital, Boston, MA, USA. '3niversity of Pittsburgh, Pittsburgh, PA, USA. ${ }^{4}$ University of Pennsylvania, Philadelphia, PA, USA. (turka@mail.med.upenn.edu)

From 15 to 19 July 2002, the first FASEB Summer Research Conference devoted to the topic of transplantation immunology was held at the Vermont Academy (Saxton's River, VT).

For the past 50 years, since the discovery of acquired transplantation tolerance by Medawar and coworkers, the field of transplantation immunology has been a vibrant and focused discipline. Growth of this research field has occurred concurrently with that of clinical transplantation. Almost 50 years ago Murray and colleagues performed the first successful clinical renal transplant. Since then, clinical transplantation has seen tremendous growth worldwide, so that transplantation of heart, liver, pancreas and lung, among other solid organs and tissues, is now routine treatment for end-stage organ failure. Furthermore, the transplantation community conducts numerous clinical trials of pharmacologic and biologic agents each year and is at the leading edge of translational immunologic research. Indeed many drugs commonly used at present for nontransplant indications (for example, cyclosporine and mycophenolate mofetil) were initially studied and introduced into clinical use as prophylaxis for transplant rejection.

Like many, we firmly believe that strong basic science research is the foundation of advances in the treatment of immune-mediated disorders. We also believe that the small, intimate, informal settings of meetings such as the FASEB Summer or Gordon conferences are ideal for free and open discussion, presentation of new data and initiation of new collaborations. Only one Keystone meeting, organized several years ago by Jeff Platt and Kathryn Wood, has focused on transplantation. What has evolved instead are meetings focused on tolerance or lymphocyte activation that devote a small number of individual talks to the topic of transplantation. Although helpful and appreciated, these sessions are inevitably unable to accommodate the large amount of new research in the field and also do not attract trainees in transplantation, whose presence and interaction with senior scientists adds so much to the character of meetings.
To try to address this deficiency, a FASEB conference devoted to the topic of transplantation immunology was organized. Our goals were similar to those of organizers of other such meetings: to provide a venue for scientists at all levels (including a significant number from outside

North America) to present new work, debate ideas with each other and make new friends and colleagues. An additional goal, however, was to deliberately include many scientists outside the immediate field of transplantation in an effort to cross-fertilize our work and theirs. It was hoped that research by established transplant immunologists would be broadened and enriched, established nontransplant investigators could be recruited into the field, and students and postdoctoral fellows interested in transplantation would be stimulated to think "outside the box". To accomplish this, roughly one-third of the invited speakers were scientists who are not considered transplant immunologists. Most of these individuals are basic researchers, whose laboratories focus on areas of fundamental importance to transplantation biology, such as T cell-APC interactions, dendritic cells, innate immunity and leukocyte adhesion. What evolved surpassed our expectations: synergy developed between basic and clinical scientists that clearly benefited both groups. Over $90 \%$ of those attending believed this was among the top $10 \%$ of meetings they had attended, and every attendee believed the conference should be repeated on a regular basis.

We are currently in the process of organizing a meeting for 2004 and plan on following the same design. This type of approach would be useful for other disciplines within the broader field of immunology. In particular, fields such as cancer immunology, infectious diseases and parasitology — with a strong translational component-seem amenable to this concept. In this age of everenlarging scientific meetings, there are far too few opportunities for in-depth interactions among scientists working in related and complementary areas. We believe that bringing together basic and applied scientists to focus on a disease process should be the pattern for the future. FASEB Summer Research meetings, and other similar venues, provide an opportunity for this to happen, and it is up to the scientific community's leaders to take advantage of it. 